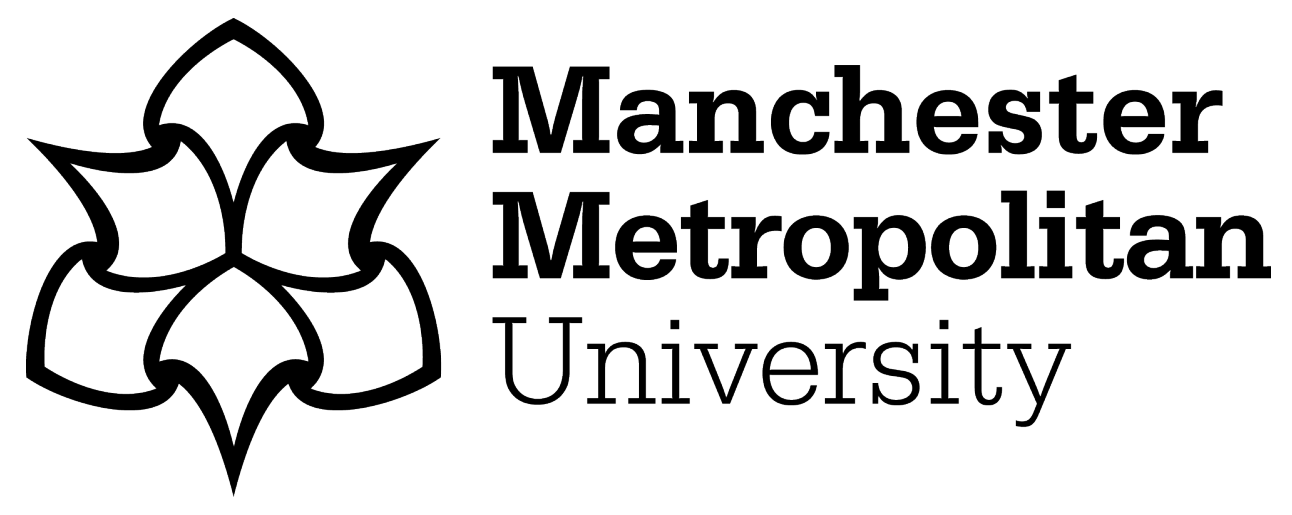

Chow, KK (2017) Low pump threshold CVD graphene based passively harmonic mode-locked fibre laser. Electronics Letters, 53 (5). pp. 330-331. ISSN 0013-5194

Downloaded from: https://e-space.mmu.ac.uk/617984/

Version: Accepted Version

Publisher: Institution of Engineering and Technology

DOI: https://doi.org/10.1049/el.2016.4325

Please cite the published version 


\section{Low pump threshold CVD graphene based passively harmonic mode-locked fibre laser}

\section{Kin Kee Chow ${ }^{\infty}$}

A low pump threshold passively harmonic mode-locked fibre laser is demonstrated. The key component is a chemical vapour deposition grown graphene saturable absorber to enable higher order harmonic mode-locking (HML) of the fibre laser with good output noise characteristics. With a relatively low pump power of $100 \mathrm{~mW}$, the fibre laser generates $\sim 1$ ps soliton pulses at the 21 st order of HML with $40 \mathrm{~dB}$ of super-mode noise suppression.

Introduction: Ultra-short optical pulse generation with high repetition rates from graphene-based passively mode-locked fibre lasers have attracted much research attention, in view of their potential applications in many disciplines, such as optical frequency metrology and high speed optical communication [1-3]. To get high repetition rates, one effective approach is to decrease the laser cavity length to the lowest possible extent [4]. However, it has practical limitations such as accessible levels of rare-earth doping of the gain fibre and practical lengths of the fibre components. Another attractive way by harmonic mode-locking (HML) mechanism, where the fundamental repetition rate might be multiplied by a significant factor [5]. In HML fibre lasers, when the pump power is increased beyond a certain value, splitting of the single pulse into multiple pulses in the cavity could occur due to the energy quantisation effect of the soliton. Normally the generated multiple pulses are randomly located. However, under specific cavity setting conditions, these multiple pulses are possible to be self-arranged to form a stable pulse train with a multiplied repetition rate corresponding to the fundamental frequency of the laser cavity.

HML in erbium-doped fibre (EDF) lasers employing graphene as the SA has been previously reported $[1,2]$. In HML lasers, many supermodes oscillate simultaneously competing with each other and the superposition of these oscillating super-modes is known as super-mode noise. This noise gives rise to unequal distribution of energies among the generated optical pulses and results in amplitude fluctuations of the output pulses. In particular, the highest repetition rate of $2.22 \mathrm{GHz}$ (21st harmonic) with $40 \mathrm{~dB}$ supermode noise suppression (SNS) by using mechanically exfoliated graphene-based SA has been examined [1]. In general, high pump thresholds are required to generate HML but recent report shows that HML can be achieved even with low pump powers using graphene as SA [6]. Besides the low pump threshold, it is crucial to obtain high SNS, which however has not been previously characterised. Incidentally, most of the HML work has been focused on the high repetition rate, without significant mention about the pulse energy fluctuations in their lasers $[1,2,6]$. It is worth noting that the pulse energy and/or amplitude instability is one of the key issues and it must be considered before the mode-locked EDF lasers can be considered for practical applications [7].

In this Letter, we experimentally demonstrate a passively HML fibre laser with a relatively low pump threshold using chemical vapour deposition (CVD) grown graphene as saturable absorber. The laser could operate either at a fundamental frequency or a higher harmonic depending on the pump power level. The fibre laser can operate up to the 21 st order of HML by adjusting the pump power and the state of polarisation in the cavity. The fibre laser not only has a low pump threshold, but its SNS is also as high as $40 \mathrm{~dB}$. In addition, the output RF spectrum has been analysed to prove that the fibre laser can achieve HML with less pulse energy fluctuations. Such passively HML fibre laser with relatively low pumping threshold, high SNS and low pulse-to-pulse fluctuations can be useful in important applications such as optical sampling, laser ranging, or ultra-precise micromachining

Experiment and results analysis: The graphene-based SA is prepared by cold transferring a piece of free standing CVD graphene film onto the end facet of a FC/APC fibre connector. The SA device is then formed by connecting another FC/APC fibre connector with an adaptor. The total insertion loss of the graphene SA is about $1.6 \pm 0.1 \mathrm{~dB}$. The saturable absorption properties of the graphene SA is then characterised using a convention mode-locked fibre laser emitting $\sim 400 \mathrm{fs}$ pulses at $1562.12 \mathrm{~nm}$, with a repetition rate of $67.1 \mathrm{MHz}$ and an average output power of $\sim 7 \mathrm{~mW}$. The modulation depth and non-saturable losses of the graphene SA are measured to be $\sim 2.5$ and $\sim 30 \%$, respectively.
The experimental setup of the graphene HML fibre laser is illustrated in Fig. 1. The laser uses a 70-cm long EDF (nLight LIEKKI Er110-4/125) as the gain medium and pumped at $974 \mathrm{~nm}$ by a laser diode delivering up to $300 \mathrm{~mW}$ coupled through a $980 / 1550$ wavelength division multiplexing (WDM) coupler. The core absorption of the EDF is $110 \mathrm{~dB} / \mathrm{m}$ at $1530 \mathrm{~nm}$ with a group velocity dispersion of $0.012 \mathrm{ps}^{2} / \mathrm{m}$. To ensure unidirectional operation, a polarisation independent isolator is placed after the SA, while the state of polarisation in the laser cavity is adjusted by an in-line fibre polarisation controller (PC). A 30/70 fused fibre optical coupler is used to extract $30 \%$ of the power from the cavity as the laser output. The total cavity length is $11.7 \mathrm{~m}$, including the standard single mode fibre pigtails with a total length of $11 \mathrm{~m}$ and a group velocity dispersion coefficient of $18(\mathrm{ps} / \mathrm{nm}) / \mathrm{km}$. The net dispersion of the laser cavity is estimated to be $-0.269 \mathrm{ps}^{2}$. The output pulses are monitored by an optical spectrum analyser with a resolution of $0.1 \mathrm{~nm}$, an optical autocorrelator (Alnair Labs HAC-200), and a $1 \mathrm{GHz}$ oscilloscope together with a $5 \mathrm{GHz}$ photodetector (Thorlabs SIR5-FC). Self-starting of mode-locked operation at fundamental frequency is found readily obtained by increasing the pump power beyond a mode-locking threshold of $26 \mathrm{~mW}$ with appropriately adjusted polarisation. The output pulse repetition rate is measured to be $\sim 17.1 \mathrm{MHz}$ which corresponds to the cavity round-trip time, confirming that the laser operates at its fundamental frequency. The measured output power is at the level of $0.3 \mathrm{~mW}$.

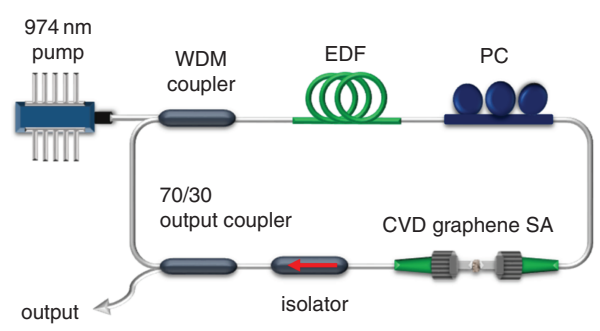

Fig. 1 Experimental setup of the passively $H M L$ fibre laser with CVD graphene SA. WDM, wavelength-division multiplexing; EDF, EDF; $P C$, polarisation controller; $C V D, C V D ; S A$, saturable absorber

Fig. $2 a$ shows a typical optical spectrum with Kelly sidebands, confirming that the fibre laser operated in the soliton regime with net-anomalous dispersion. The centre wavelength and full-width halfmaximum (FWHM) are found to be 1567.4 and $2.23 \mathrm{~nm}$, respectively. The autocorrelation trace of the output pulse is depicted in Fig. $2 b$, showing that the pulse duration is $\sim 1.13$ ps. With a $\operatorname{sech}^{2}$ pulse fitting, the calculated time-bandwidth product (TBP) of the output pulses is 0.31 , which is near to transform-limit (0.315).
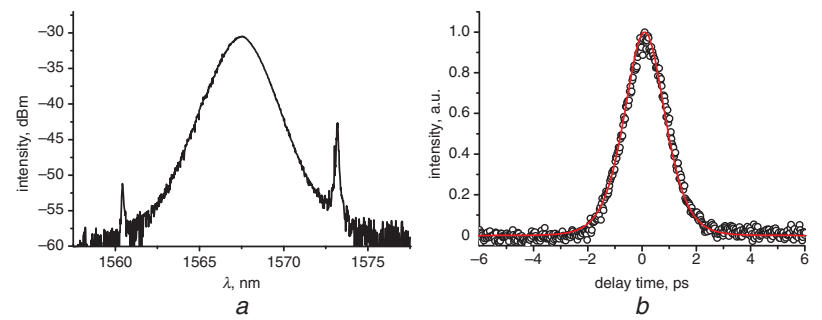

Fig. 2 Laser output at the fundamental frequency

$a$ Optical spectrum

$b$ Autocorrelation trace

With further increasing the pump power and adjusting the PC, multiple pulses are generated due to the energy quantisation effect. At the beginning, the pulses are randomly distributed in the cavity. However, by carefully adjusting the PC, we are able to obtain stable output single pulses with a multiplied repetition rate corresponding to the fundamental frequency of the laser cavity. By carefully increasing the pump power from 30 to $100 \mathrm{~mW}$, the harmonic order of the modelocked pulses is found changing from the 2 nd to the 21 st order. When the pump power reaches $100 \mathrm{~mW}$, HML at the $21 \mathrm{st}$ harmonic of $\sim 360 \mathrm{MHz}$ corresponding to the fundamental frequency is observed. The harmonic order decreases stepwise as the pump power decreases, where the polarisation of laser cavity is slightly adjusted for operation 
of each harmonic order. Once passively HML is established, the fibre laser is found stably operating.

Fig. $3 a$ shows the output spectrum of the 21 st harmonic, which is centred at $1567.6 \mathrm{~nm}$ with a FWHM of $2.55 \mathrm{~nm}$. Fig. $3 b$ further depicts the autocorrelation trace of the output pulses with $\operatorname{sech}^{2}$ fitting. The output pulse width is measured to be around $1 \mathrm{ps}$. Taking into account of the spectral bandwidth, the TBP of the laser is estimated to be 0.31 , which is close to the transform limit for $\operatorname{sech}^{2}$-fitting.
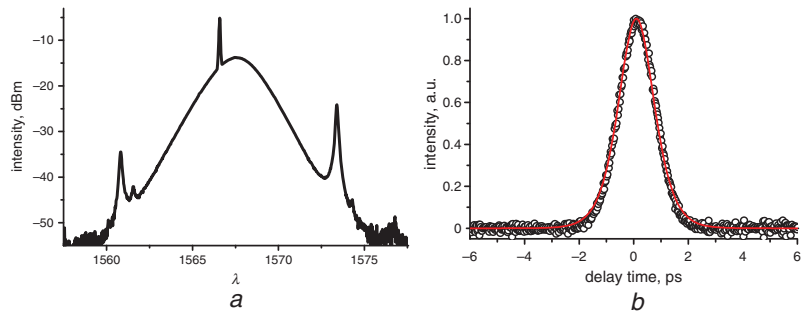

Fig. 3 Laser output at the 21st HML

a Optical spectrum

$b$ Autocorrelation trace

Fig. 4 further plots the RF spectra of the fundamental and the $21 \mathrm{st}$ HML over $80 \mathrm{kHz}$ frequency span with a bandwidth resolution of $200 \mathrm{~Hz}$. The RF spectrum shows a peak-to-pedestal ratio of $\sim 53 \mathrm{~dB}$ for fundamental and $\sim 56 \mathrm{~dB}$ for $21 \mathrm{st}$ harmonic, respectively. The energy instability of the generated pulses is investigated via the RF spectrum. Fluctuations in the pulse energy can be estimated from the fundamental component of the RF spectrum by [8]:

$$
\frac{\Delta E}{E}=\sqrt{\left(\frac{\Delta P \Delta f}{\Delta f_{\mathrm{res}}}\right)}
$$

where $\Delta P$ is the power ratio between the signal and the peak of the noise band, $\Delta f$ is the frequency width of the noise floor, and $\Delta f_{\text {res }}$ is the spectral resolution of the spectrum analyser. For fundamental frequency component, with $\Delta P=10^{-5.3}, \Delta f=3.7 \mathrm{kHz}$ and $\Delta f_{\text {res }}=200 \mathrm{~Hz}$, the pulse energy fluctuation is estimated to be $0.95 \%$. Likewise, the pulse-to-pulse energy fluctuations calculated for the 21 st harmonic is only $0.74 \%$, with $\Delta P=10^{-5.6}, \Delta f=4.3 \mathrm{kHz}$ and $\Delta f_{\text {res }}=200 \mathrm{~Hz}$.
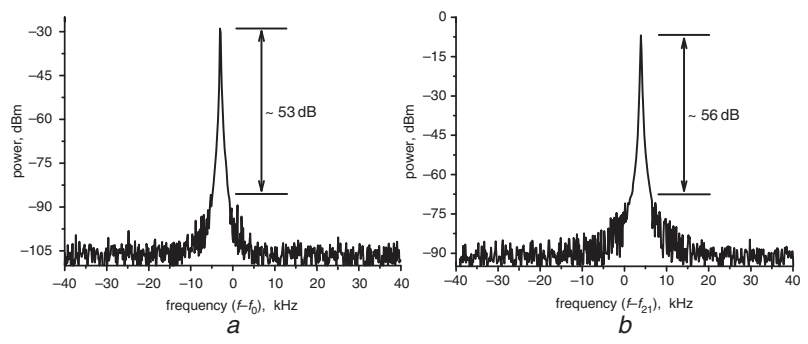

Fig. 4 RF spectra of laser output

$a$ Fundamental $\left(f_{0} \sim 17.14 \mathrm{MHz}\right)$, and

$b$ 21st harmonic $\left(f_{21} \sim 360 \mathrm{MHz}\right)$ with $200 \mathrm{~Hz}$ resolution
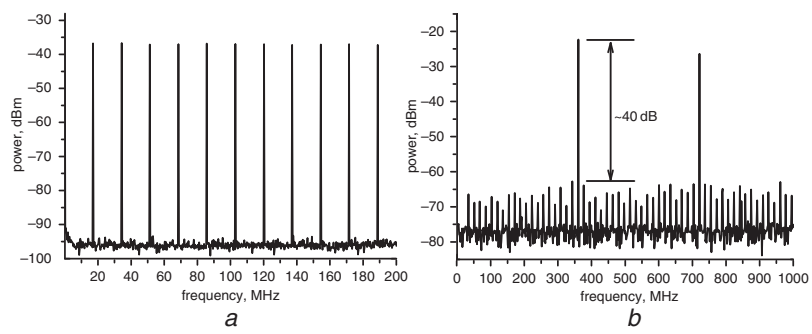

Fig. 5 RF spectrum of the laser mode-locked at

$a \sim 17.14 \mathrm{MHz}$ with $200 \mathrm{MHz}$ span

$b \sim 360 \mathrm{MHz}$ with $1 \mathrm{GHz}$ span
A steady train of cavity harmonics without sidebands is shown Fig. $5 a$, suggesting good pulse-train stability and no Q-switching instabilities. Fig. $5 b$ shows the RF spectrum of the 21 st harmonic recorded with a span of $1 \mathrm{GHz}$ and resolution bandwidth of $10 \mathrm{kHz}$. The results show a SNS as high as $40 \mathrm{~dB}$, which is similar to that of the previously reported HML fibre lasers [1]. To further evaluate the significance of the SA in achieving the HML results, we replace the CVD-grown graphene based SA and with a liquid-phase exfoliation based graphene SA [3]. No HML is observed with the liquid-phase exfoliation prepared graphene based SA when applying to the same laser cavity. Therefore, it is believed that the CVD-grown graphene plays a significant role to achieve HML operation and subsequently lead to the high SNS and the less energy fluctuations of the generated pulses.

Conclusion: We demonstrated a relatively low pump power HML EDF laser by employing a CVD graphene SA. Under different launched pump powers and appropriate adjustment of polarisation in the laser cavity, HML up to 21 st order has been obtained. A relatively low pump power of $100 \mathrm{~mW}$ enables the generation of the 21st HML pulses at $360 \mathrm{MHz}$ repetition rate with $40 \mathrm{~dB}$ of super-mode noise suppression. The RF spectrum data analysis demonstrates that the laser emits high-quality pulses with low energy fluctuations. The results confirm that the CVD-grown graphene can be used in fibre lasers for low pump threshold high-order passive HML operation and generating output pulses with high super-mode noise suppression and low pulse-to-pulse energy fluctuations.

(C) The Institution of Engineering and Technology 2017

Submitted: 25 November 2016

doi: $10.1049 /$ el.2016.4325

One or more of the Figures in this Letter are available in colour online.

Kin Kee Chow (School of Engineering, Manchester Metropolitan University, John Dalton Building, Chester Street, Manchester M1 5GD, $U K)$

凶 E-mail: k.chow@mmu.ac.uk

\section{References}

1 Sobon, G., Sotor, J., and Abramski, K.M.: 'Passive harmonic modelocking in Er-doped fiber laser based on graphene saturable absorber with repetition rates scalable to $2.22 \mathrm{GHz}$, Appl. Phys. Lett., 2012, 100, p. 161109

2 Meng, Y.C., Zhang, S.M., Li, X.L., Li, H.F., Du, J., and Hao, Y.P.: 'Multiple-soliton dynamic patterns in a graphene mode-locked fiber laser', Opt. Express, 2012, 20, pp. 6685-6692

3 Mamidala, V., Woodward, R.I., Yang, Y., Liu, H.H., and Chow, K.K.: 'Graphene-based passively mode-locked bidirectional fiber ring laser', Opt. Express, 2014, 22, pp. 4539-4546

4 Nicholson, J.W., and DiGiovanni, D.J.: 'High-repetition-frequency lownoise fiber ring lasers mode-locked with carbon nanotubes', IEEE Photonic Tech. Lett., 2008, 20, pp. 2123-2125

5 Zhu, X., Wang, C., Liu, S., and Zhang, G.: 'Tunable high-order harmonic mode-locking in Yb-doped fiber laser with all-normal dispersion', IEEE Photonic Tech. Lett., 2012, 24, pp. 754-756

6 Meng, Y.C., Zhang, S.M., Li, X.L., Li, H.F., Du, J., and Hao, Y.P.: 'Passive harmonically mode-locked fiber laser with low pumping power based on a graphene saturable absorber', Laser Phys. Lett., 2012, 9, pp. 537-541

7 Zhao, D., Lai, Y., Shu, X., Zhang, L., and Bennion, I.: 'Supermode-noise suppression using a nonlinear Fabry-Perot filter in a harmonically modelocked fiber ring laser', Appl. Phys. Lett., 2002, 81, pp. 4520-4522

8 Vonderlinde, D.: 'Characterization of the noise in continuously operating mode-locked lasers', Appl. Phys. B, 1986, 39, pp. 201-217 